\title{
ON THE STRUCTURE OF A MORSE FORM FOLIATION
}

\author{
I. Gelbukh, Mexico City
}

(Received May 6, 2007)

\begin{abstract}
The foliation of a Morse form $\omega$ on a closed manifold $M$ is considered. Its maximal components (cylinders formed by compact leaves) form the foliation graph; the cycle rank of this graph is calculated. The number of minimal and maximal components is estimated in terms of characteristics of $M$ and $\omega$. Conditions for the presence of minimal components and homologically non-trivial compact leaves are given in terms of $\mathrm{rk} \omega$ and Sing $\omega$. The set of the ranks of all forms defining a given foliation without minimal components is described. It is shown that if $\omega$ has more centers than conic singularities then $b_{1}(M)=0$ and thus the foliation has no minimal components and homologically non-trivial compact leaves, its folitation graph being a tree.
\end{abstract}

Keywords: number of minimal components, number of maximal components, compact leaves, foliation graph, rank of the form

MSC 2000: 57R30, 58K65

\section{INTRODUCTION AND ANNOUNCEMENT OF THE RESULTS}

Consider a connected closed oriented manifold $M$ with a Morse form $\omega$, i.e., a closed 1-form with Morse singularities - locally the differential of a Morse function. The set of its singularities Sing $\omega$ is finite. This form defines a foliation $\mathcal{F}_{\omega}$ on $M \backslash \operatorname{Sing} \omega$. Its leaves $\gamma$ can be classified into compact, compactifiable $(\gamma \cup \operatorname{Sing} \omega$ is compact), and non-compactifiable.

Such foliations have remarkably regular structure. A connected component $\mathcal{C}_{i}^{\text {max }}$ of the union of compact leaves - which we call maximal component - is an open cylinder over any its leaf, whose levels are leaves. In particular, all leaves in a maximal component are diffeomorphic. A connected component $\mathcal{C}_{i}^{\text {min }}$ of the union of noncompactifiable leaves is called minimal component. Its topology can be arbitrarily complex — say, such a component can cover the whole $M \backslash \operatorname{Sing} \omega$ [1] - but it cannot be too simple: a minimal component contains at least two cycles with noncommensurable integrals [10]. Each non-compactifiable leaf is dense in its minimal component [7]. The boundary of a maximal or minimal component consists of a finite number of non-compact compactifiable leaves $\gamma_{i}^{0}$ and singularities. This gives

Cite this paper as: Czechoslovak Mathematical Journal, 59(1):207-220, 2009. Pre-print version. Final version: http://dx.doi.org/10.1007/s10587-009-0015-5 
a decomposition of $M$ into a finite number of non-intersecting pieces

$$
M=\left(\bigcup \mathcal{C}_{i}^{\max }\right) \cup\left(\bigcup \mathcal{C}_{i}^{\text {min }}\right) \cup\left(\bigcup \gamma_{i}^{0}\right) \cup \operatorname{Sing} \omega,
$$

interconnected in a way resembling the structure of a simplicial complex.

The way the pieces are put together is described by the foliation graph $\Gamma-\mathrm{a}$ connected graph (allowing loops and multiple edges) whose edges are $\mathcal{C}_{i}^{\text {max }}$ and vertices are connected components of $M \backslash \cup \mathcal{C}_{i}^{\max }$. This notion has been used for studying foliation structure $[2,6]$. Note that unlike [10], we define the graph on the whole $M$, including Sing $\omega$.

In this paper, we show (Theorem 2.1) that

$$
m(\Gamma)=c(\omega),
$$

where $m(\Gamma)$ is the cycle rank of the foliation graph $\Gamma$ and $c(\omega)$ is the number of homologically independent compact leaves of $\mathcal{F}_{\omega}$.

The number $m(\omega)$ of minimal components is bounded by the first Betti number: $2 m(\omega) \leq b_{1}(M)[1,8]$. We obtain a stronger estimate (Theorem 3.1):

$$
2 m(\omega)+c(\omega) \leq b_{1}(M)
$$

and an independent estimate (our main theorem, Theorem 3.2):

$$
m(\omega)+c(\omega) \leq h(M),
$$

where $h(M)$ is the maximum rank of a subgroup in $H^{1}(M, \mathbb{Z})$ with trivial cupproduct [11]. There are practical methods of calculating $h(M)$ (Remark 3.1).

We also estimate (Theorem 3.3) the total number of components for a singular form:

$$
M(\omega)+m(\omega) \leq h(M)+|\operatorname{Sing} \omega|-1
$$

where $M(\omega)$ is the number of maximal components (obviously, for a non-singular form $M(\omega)+m(\omega)=1$ instead). In addition, for a singular form

$$
M(\omega)+2 m(\omega) \leq b_{1}(M)+|\operatorname{Sing} \omega|-1 .
$$

In addition to the bound for $m(\omega)+c(\omega)$ above, we present some conditions for $m(\omega)=0$ and for $c(\omega)=0$. A foliation having no minimal components is called compactifiable. In [3] we have presented some conditions for compactifiability of the foliation in terms of the structure of $\operatorname{ker}[\omega] \subseteq H_{1}(M)$, where $[\omega]$ is the integration map. Here we consider other characteristics of the form: the form's rk $\omega \stackrel{\text { def }}{=} \operatorname{rkim}[\omega]$ and the number of singularities of different indices.

If $\omega$ is rational $(\operatorname{rk} \omega \leq 1)$ then $\mathcal{F}_{\omega}$ is compactifiable [13]. We show that the converse is true only in the sense that any compactifiable foliation can be defined by a rational Morse form. Namely, a compactifiable foliation $\mathcal{F}_{\omega}$ is defined by forms $\omega^{\prime}$ with

$$
(0 \text { or } 1) \leq \operatorname{rk} \omega^{\prime} \leq c(\omega)
$$

(Theorem 4.1); whether 0 is included depends on the structure of the directed foliation graph [4]. In particular, if $\operatorname{rk} \omega>h(M)$ then $\mathcal{F}_{\omega}$ is not compactifiable. For instance, if $\operatorname{rk} \omega=b_{1}(M)$ (i.e. maximal) and the cup-product on $H^{1}(M, \mathbb{Z})$ is nontrivial then $\mathcal{F}_{\omega}$ is non-compactifiable (Corollary 4.3). If in addition $H_{1}(M)$ has no 
torsion and the cup-product is non-degenerate then all compact leaves of $\mathcal{F}_{\omega}$ are homologically trivial (Proposition 4.1).

No necessary condition for compactifiability of $\mathcal{F}_{\omega}$ can be obtained in terms of Sing $\omega$ : for any foliation there exists a rational Morse form with the same set of singularities of each index [14]. However, Sing $\omega$ can give useful information on $M$. If Sing $\omega=\emptyset$ then $M$ is a bundle over $S^{1}$ [15], so topology of $\mathcal{F}_{\omega}$ is defined by rk $\omega$ : if $\mathrm{rk} \omega=1$ then $\mathcal{F}_{\omega}$ is compact (all leaves are compact), otherwise it is minimal. If all singularities are centers then $M=S^{n}$ and $\mathcal{F}_{\omega}$ is obviously compact. (By centers, called also spherical singularities, we mean those of index 0 or $\operatorname{dim} M$.)

We generalize the latter fact: If there are more centers than conic singularities then (Theorem 4.2)

$$
b_{1}(M)=0
$$

in particular, in this case $m(\omega)+c(\omega)=0$, i.e., $\mathcal{F}_{\omega}$ is compactifiable, all its leaves are homologically trivial, and its foliation graph is a tree (Theorem 4.3).

The paper is organized as follows. In Section 2 we remind some facts about qualitative structure of a Morse form foliation and give necessary definitions. We introduce minimal and maximal foliation components and describe their properties (Section 2.1). Then we define the foliation graph and calculate its cycle rank (Section 2.2). In Section 3 we give the upper bounds on the number of minimal components (Section 3.1) and on the total number of (minimal and maximal) components of the foliation (Section 3.2). Finally, in Section 4 we give some conditions on the presence of minimal components in terms of the rank of the form (Section 4.1) and the indices of its singularities (Section 4.2).

\section{General structure}

The general structure of a Morse form foliation has been studied in $[1,3,7,10]$.

Definition 2.1 ([3]). A leaf $\gamma \in \mathcal{F}_{\omega}$ is called compactifiable if $\gamma \cup \operatorname{Sing} \omega$ is compact; otherwise it is called non-compactifiable.

Note that compact leaves are compactifiable.

The set covered by all non-compactifiable leaves is open [7]. A compact leaf $\gamma$ has an open neighborhood consisting solely of compact leaves [1,2]: indeed, integrating $\omega$ gives $f$ with $d f=\omega$ near $\gamma$; hence the set covered by all compact leaves is also open. The number of non-compact compactifiable leaves $\gamma_{i}^{0}$ is finite since each singularity belongs to the closure of no more than four non-compact compactifiable leaves (see Figure 1); thus such leaves are isolated.

2.1. Minimal and maximal foliation components. We call foliation components connected components $\mathcal{C}_{i}$ of the set $M \backslash\left(\bigcup \gamma_{i}^{0} \cup \operatorname{Sing} \omega\right)$, i.e. the union of all compact and non-compactifiable leaves. Each component $\mathcal{C}_{i}$ is open, $\partial \mathcal{C}_{i} \subseteq \cup \gamma_{j}^{0} \cup \operatorname{Sing} \omega$. For any $\gamma_{j}^{0}$ there exist either one or two components $\mathcal{C}_{i}$ such that $\gamma_{j}^{0} \cap \partial \mathcal{C}_{i} \neq \emptyset$; see Figure 2(a). Thus the number of components is finite.

A component consists entirely of leaves of one type: either non-compactifiable or compact. Components of the former kind are minimal neighborhoods consisting of non-compactifiable leaves; each such leaf is dense in its minimal component $[1,7]$ : 


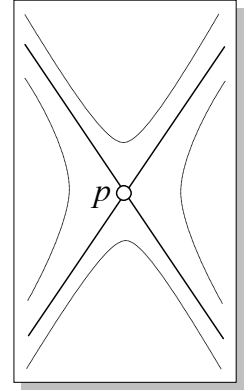

(a)

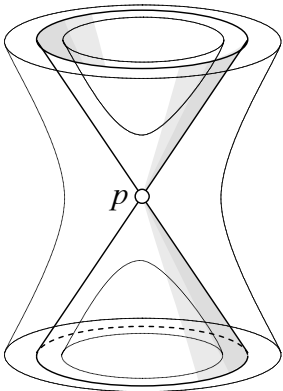

(b)

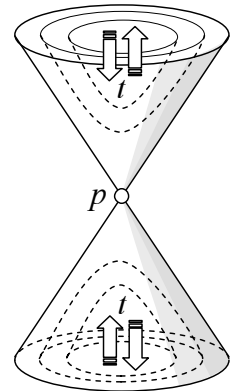

(c)

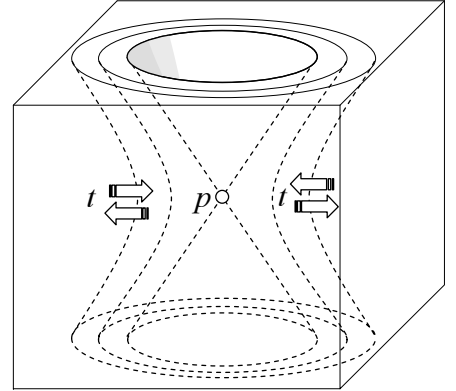

(d)

FiguRE 1. A conic singularity has locally four $(a)$ or two $(b),(c)$ adjacent (non-compact) leaves; centers have no such leaves, and other singularities have one $(d)$. Here, $(d)$ and $(c)$ visualize non-compact leaves for $\operatorname{dim} M=4$ (the lowest dimension where singularities other than conic or centers exist) as connected components of the set $\left\{\mathbb{R}^{4} \backslash p \mid x^{2}+y^{2}-z^{2}= \pm t^{2}\right\}$, respectively, foliated by the "time" $t$.

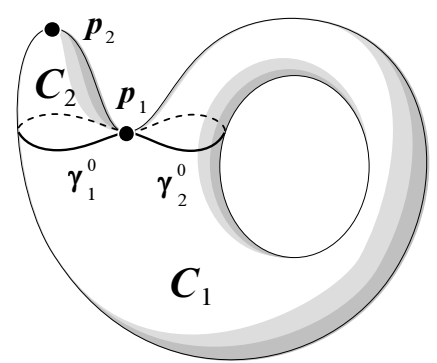

(a)

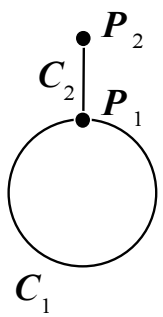

(b)

Figure 2. (a) The leaf $\gamma_{2}^{0}$ adjoins only one component, while $\gamma_{1}^{0}$ connects two components. (b) The corresponding foliation graph has a loop.

Definition 2.2 ([1])). A connected component of the union of all non-compactifiable leaves is called a minimal component of the foliation.

Components of the latter kind are maximal connected sets consisting of compact leaves:

Definition 2.3 ([3]). A connected component of the union of all compact leaves is called a maximal component of the foliation. 
A maximal component $\mathcal{C}$ of a singular Morse form foliation is cylindrical: $\mathcal{C}=$ $\gamma \times(0,1), \gamma \times x \in \mathcal{F}_{\omega}$, where $\gamma$ is any (compact) leaf in $\mathcal{C}$. Note that for a nonsingular form a maximal component - which exists iff $\omega$ is rational - is a bundle over $S^{1}$ with fiber $\gamma \in \mathcal{F}_{\omega}$ and covers the whole manifold [15].

While a maximal component is topologically simple, the topology of a minimal component can be arbitrarily complex (e.g., it can cover the whole $M \backslash \operatorname{Sing} \omega$ [1]). Moreover, it cannot be too simple: a minimal component contains at least two (homologically independent in $M$ ) 1-cycles with incommensurable periods [10].

Thus $M$ can be decomposed into a finite number of non-intersecting pieces interconnected in a way resembling the structure of a simplicial complex:

$$
M=\left(\bigcup \mathcal{C}_{i}^{\max }\right) \cup\left(\bigcup \mathcal{C}_{j}^{\text {min }}\right) \cup\left(\bigcup \gamma_{k}^{0}\right) \cup \operatorname{Sing} \omega,
$$

with maximal components $\mathcal{C}_{i}^{\max }$ being cylindrical and minimal components $\mathcal{C}_{j}^{\text {min }}$ having at least two independent 1-cycles. For examples of such a decomposition see Figure $2(a), 3(a, c)$, and $4(b)$.

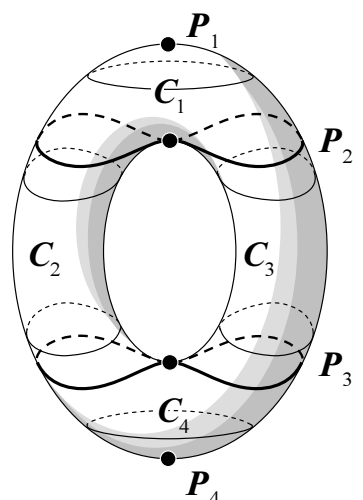

(a)

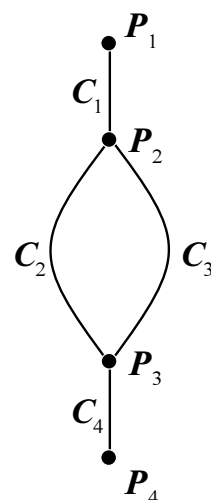

(b)

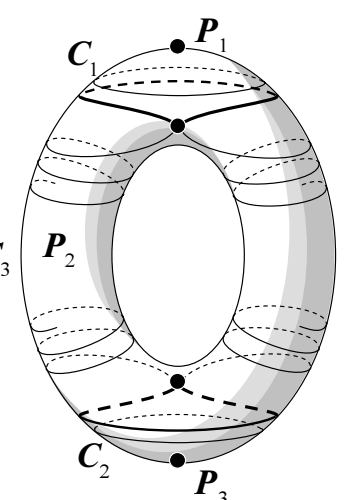

(c)

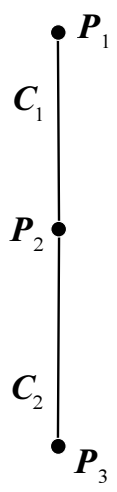

(d)

Figure 3. (a), (c) Examples of the decomposition. (b) The vertices of $\Gamma$ can include singularities, non-compact compactifiable leaves and $(d)$ whole minimal components.

2.2. Foliation graph. The configuration formed by the maximal components in the decomposition (2.1) is described by the foliation graph [3]. Rewrite (2.1) as

$$
M=\left(\bigcup \mathcal{C}_{i}^{\max }\right) \cup\left(\bigcup P_{j}\right),
$$

where $P_{j}$ are connected components of the union $P$ of all non-compact leaves and singularities. Since a maximal component is a cylinder, $\partial \mathcal{C}_{i}^{\max } \subseteq P$ consists of one or two connected components; thus each $\mathcal{C}_{i}^{\max }$ adjoins one or two of $P_{j}$. This allows representing $M$ as a connected graph $\Gamma$ (admitting multiple edges and loops) with edges $\mathcal{C}_{i}^{\max }$ and vertices $P_{j}$ : an edge $\mathcal{C}_{i}^{\max }$ is incident to a vertex $P_{j}$ if $\partial \mathcal{C}_{i}^{\max } \cap P_{j} \neq \emptyset$; see Figure 2 and 3. 
Let $c(\omega)=\operatorname{rk} H_{\omega}$, where $H_{\omega} \subseteq H_{n-1}(M)$ is generated by all compact leaves, i.e., the number of homologically independent compact leaves. Denote by $m(\Gamma)$ the cycle rank of the graph $\Gamma$.

Theorem 2.1. It holds

$$
c(\omega)=m(\Gamma) .
$$

Moreover, there exist $\gamma_{1}, \ldots, \gamma_{c(\omega)}$ such that for any compact leaf $\gamma$ it holds $[\gamma]=$ $\sum_{i \in I_{\gamma}} \pm\left[\gamma_{i}\right]$.

Proof. In $H_{\omega}$ there exists a basis $e$ consisting of homology classes of leaves $\left[\gamma_{1}\right], \ldots,\left[\gamma_{c}\right]$ $c=c(\omega)[3]$. Each $\gamma_{i}, i=1, \ldots, c$, defines a maximal component $\mathcal{C}_{i}^{\max }=\gamma_{i} \times(0,1)$, which is an edge $h_{i}$ in the foliation graph $\Gamma$; see Figure 4(a).

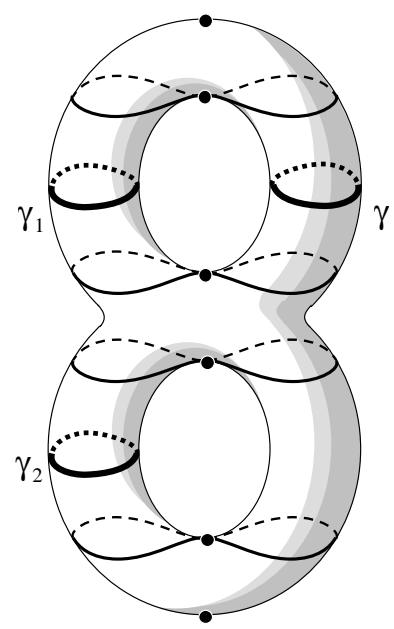

(a)

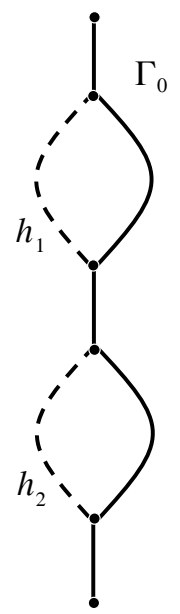

(b)

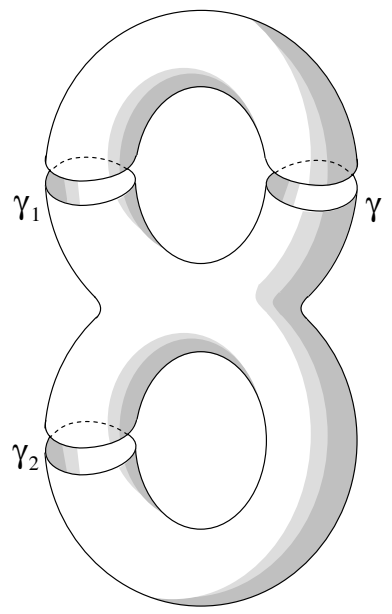

(c)

Figure 4. Proof of Theorem 2.1.

Consider the subgraph $\Gamma^{0}=\Gamma \backslash \cup_{i=1}^{c} h_{i}$; see Figure 4(b). It has the same set of vertices. Independence of $e$ implies that $\Gamma^{0}$ is connected.

Suppose that $\Gamma^{0}$ contains a cycle $z$; associate with it a curve $\alpha \subset M$. Obviously, $\alpha \cap \gamma_{i}=\emptyset$ for all $i$. Let $h \in z$ be an edge, and $\gamma \subset M$ a corresponding leaf. Then $[\alpha] \cdot[\gamma] \neq 0$ and $[\alpha] \cdot\left[\gamma_{i}\right]=0$, which contradicts maximality of $e$.

Thus $\Gamma^{0}$ is a spanning tree. Since the cycle rank of a graph equals to the number of chords of its spanning tree [5], we have $m(\Gamma)=c(\omega)$. What is more, since removing any edge splits a tree, for any compact leaf $\gamma$ we have $[\gamma]=\sum_{i \in I_{\gamma}} \pm\left[\gamma_{i}\right]$; see Figure 4(c).

Corollary 2.1. If all compact leaves of $\mathcal{F}_{\omega}$ are homologically trivial then $\Gamma$ is a tree. If in addition $\mathcal{F}_{\omega}$ has no minimal components then $\omega=d f$.

The second statement follows from Corollary 4.1 below. 


\section{NuMBER OF COMPONENTS}

Given a specific $M$, we can estimate the number of components of Morse form foliations on $M$. This may give useful information on their structure.

3.1. Number of minimal components. Denote by $m(\omega)$ the number of minimal components of $\mathcal{F}_{\omega}$. It is known that $2 m(\omega) \leq b_{1}(M)[1,8]$. We generalize this estimate:

Theorem 3.1. $2 m(\omega)+c(\omega) \leq b_{1}(M)$.

Proof. Let $z_{1}, \ldots, z_{c(\omega)}$ be 1-cycles dual to homologically independent compact leaves $\gamma_{1}, \ldots, \gamma_{c(\omega)}$, i.e., $z_{i} \cdot\left[\gamma_{j}\right]=\delta_{i j}$. Each minimal component $\mathcal{C}_{i}^{\text {min }}$ contains two cycles $z_{i}^{\prime}, z_{i}^{\prime \prime}$ with incommensurable periods [10]. Suppose

$$
\sum_{i=1}^{m(\omega)}\left(n_{i}^{\prime} z_{i}^{\prime}+n_{i}^{\prime \prime} z_{i}^{\prime \prime}\right)+\sum_{j=1}^{c(\omega)} m_{j} z_{j}=0 .
$$

Intersecting (2) with $\left[\gamma_{k}\right]$ gives $m_{k}=0$ for all $k$. Thus for any $p$ we can rewrite (3.1) as

$$
n_{p}^{\prime} z_{p}^{\prime}+n_{p}^{\prime \prime} z_{p}^{\prime \prime}=-\sum_{i \neq p}\left(n_{i}^{\prime} z_{i}^{\prime}+n_{i}^{\prime \prime} z_{i}^{\prime \prime}\right)=z .
$$

On the one hand, $z$ is induced from $\mathcal{C}_{p}^{\text {min }}$ and on the other hand, from $M \backslash \mathcal{C}_{p}^{\text {min }}$, thus by the Mayer-Vietoris sequence it is induced from $\partial \mathcal{C}_{p}^{\text {min }}$. Since $\partial \mathcal{C}_{p}^{\text {min }}$ consists of leaves and singularities, $\int_{z} \omega=0$. Thus $n_{p}^{\prime}=n_{p}^{\prime \prime}=0$, since the corresponding periods are incommensurable.

Example 3.1. For a connected sum $M=\left(S^{2} \times S^{1}\right) \sharp\left(S^{2} \times S^{1}\right)$, Theorem 3.1 gives $2 m(\omega)+c(\omega) \leq 2$. This characterizes the topology of $\mathcal{F}_{\omega}$. Indeed, if $\mathcal{F}_{\omega}$ is compactifiable, then it has at most two homologically independent compact leaves. Otherwise, it has exactly one (uniquely ergodic [1]) minimal component, and all its compact leaves are homologically trivial.

Denote by $h(M)$ the maximum rank of a subgroup in $H^{1}(M, \mathbb{Z})$ with trivial cupproduct [11].

Remark 3.1. Some methods of calculating $h(M)$ can be found in [12]:

(i) $h\left(M_{1} \times M_{2}\right)=\max \left\{h\left(M_{1}\right), h\left(M_{2}\right)\right\}$.

(ii) $h\left(M_{1} \sharp M_{2}\right)=h\left(M_{1}\right)+h\left(M_{2}\right)$ for $\operatorname{dim} M_{i} \geq 2$.

(iii) Let $b_{i}=b_{i}(M)$ be the Betti numbers and $r=\mathrm{rkker} \smile$, where $\smile$ is the cup-product on $H^{1}(M, \mathbb{Z})$. Then

$$
\frac{b_{1}+b_{2} r}{b_{2}+1} \leq h(M) \leq \frac{b_{1} b_{2}+r}{b_{2}+1} .
$$

In particular, if $b_{2}=1$ then $h(M)=\frac{1}{2}\left(b_{1}+r\right)$; if $r=b_{1}$ then $h(M)=b_{1}$.

(iv) If $\smile$ is surjective then $h(M) \leq r+\frac{1}{2}+\sqrt{\left(b_{1}-r-\frac{1}{2}\right)^{2}-2 b_{2}}$.

Example 3.2. $h\left(T^{n}\right)=1$ (torus); $h\left(M_{g}^{2}\right)=g$. 
Theorem 3.2. $m(\omega)+c(\omega) \leq h(M)$.

Proof. (i) Let us show that for a minimal component $\mathcal{C}^{\text {min }}$ there exist $u \in H_{1}\left(\mathcal{C}^{\text {min }}\right)$ and $z \in H_{n-1}\left(\mathcal{C}^{\text {min }}\right)$ such that their intersection $u \cdot z \neq 0$.

Indeed, consider the diagram

$$
\begin{aligned}
& \rightarrow H_{1}(\partial \overline{\mathcal{C}}) \stackrel{i_{*}}{\longrightarrow} \quad H_{1}(\overline{\mathcal{C}}) \quad \stackrel{j_{*}}{\longrightarrow} H_{1}(\overline{\mathcal{C}}, \partial \overline{\mathcal{C}}) \quad \rightarrow \\
& \begin{array}{cc}
\uparrow \varphi_{*} & \uparrow D \\
H_{1}(\mathcal{C}) & H^{n-1}(\mathcal{C}, \mathbb{Z})
\end{array} \\
& \uparrow D \quad \uparrow \varphi^{*} \\
& \rightarrow H^{n-1}(\overline{\mathcal{C}}, \partial \overline{\mathcal{C}}, \mathbb{Z}) \stackrel{j^{*}}{\longrightarrow} H^{n-1}(\overline{\mathcal{C}}, \mathbb{Z}) \stackrel{i^{*}}{\longrightarrow} H^{n-1}(\partial \overline{\mathcal{C}}, \mathbb{Z}) \rightarrow
\end{aligned}
$$

where $\mathcal{C}=\mathcal{C}^{\text {min }}$, the lines are exact sequences of pairs, $D$ is the Poincaré isomorphism defined by the cap-product, and homomorphisms $\varphi_{*}$ and $\varphi^{*}$ are induced by the inclusion $\varphi: \mathcal{C} \hookrightarrow \overline{\mathcal{C}}$.

Let $c \subset \mathcal{C}$ be a closed curve such that $\int_{c} \omega \neq 0$ and $u=[c]$. Then $k \varphi_{*} u \notin \operatorname{im} i_{*}$ for any $k \in \mathbb{Z}$ and $j_{*} \varphi_{*} u \neq 0$; let $\alpha=D^{-1} j_{*} \varphi_{*} u \neq 0$. Since $\alpha \in H^{n-1}(\mathcal{C}, \mathbb{Z})$ is of infinite order, it can be viewed as an element of $\operatorname{Hom}\left(H_{n-1}(\mathcal{C}), \mathbb{R}\right)$. So there exists a cycle $z \in H_{n-1}(\mathcal{C})$ such that $\alpha(z) \neq 0$. By construction, $u \cdot z \neq 0$.

(ii) For each minimal component $\mathcal{C}_{i}=\mathcal{C}_{i}^{\text {min }}$ consider the cycles $u_{i} \in H_{1}(M)$, $z_{i} \in H_{n-1}(M)$ such that $u_{i} \cdot z_{i} \neq 0$.

Consider a maximal system $\left\{\gamma_{j}\right\}$ of homologically independent compact leaves. The system $\left\{z_{i}\right\} \cup\left\{\left[\gamma_{j}\right]\right\}$ is independent. Indeed, suppose

$$
\sum p_{i} z_{i}+\sum q_{j}\left[\gamma_{j}\right]=0
$$

Since $u_{k} \cdot\left[\gamma_{j}\right]=0$ and $u_{k} \cdot z_{i}=0$ for all $i \neq k$, we have all $p_{i}=0$. Then all $q_{j}=0$ since $\left[\gamma_{j}\right]$ are linearly independent.

Finally, on $\left\{\mathcal{D} z_{i}\right\} \cup\left\{\mathcal{D}\left[\gamma_{j}\right]\right\}$, where $\mathcal{D}: H_{n-1}(M) \rightarrow H^{1}(M, \mathbb{Z})$ is the Poincaré duality, the cup-product $\smile$ is trivial, since all $\mathcal{C}_{i}$ and $\gamma_{j}$ do not intersect.

Example 3.3. For a torus $T^{n}, m(\omega)+c(\omega) \leq 1$. We have all the conclusions of Example 3.1 (with an obvious correction of its last sentence).

Remark 3.2. The bound on $m(\omega)$ implied by Theorem 3.1 and its corollary

$$
m(\omega) \leq \frac{1}{2} b_{1}(M)
$$

is independent from the bound implied by Theorem 3.2 and its corollary

$$
m(\omega) \leq h(M),
$$

as the following examples show. Which one is stronger depends on the structure of the cup-product.

Example 3.4. For a torus, we have $h\left(T^{n}\right)=1$ and $b_{1}\left(T^{n}\right)=n$. For $n=2,3$, bounds (3.3) and (3.4) are equivalent. If $n \geq 4$, even the weakened form (3.4) of Theorem 3.2 gives a stronger bound on $m(\omega)$ than Theorem 3.1. 
Example 3.5. For $M=\sharp_{i=1}^{p}\left(S^{2} \times S^{1}\right), p \geq 2$, we have $h(M)=b_{1}(M)=p$. Theorem 3.1 gives a stronger bound than Theorem 3.2 and (3.3) stronger than (3.4). Moreover, for a minimal foliation on this $M$, (3.3) gives a stronger bound than Theorem 3.2. We leave open the question of existence of manifolds on which (3.3) gives a stronger bound than Theorem 3.2 for any foliation.

3.2. Total number of components. Denote by $M(\omega)$ the number of maximal components.

Theorem 3.3. If $\operatorname{Sing} \omega \neq \emptyset$ then

$$
\begin{gathered}
M(\omega)+m(\omega) \leq h(M)+|\operatorname{Sing} \omega|-1 ; \\
M(\omega)+2 m(\omega) \leq b_{1}(M)+|\operatorname{Sing} \omega|-1 .
\end{gathered}
$$

Proof. In the foliation graph $\Gamma$ with $M(\omega)$ edges and $p$ vertices, $M(\omega)=m(\Gamma)+p-1$. Obviously, $p \leq|\operatorname{Sing} \omega|$. By Theorem 2.1 and Theorem 3.2, $m(\Gamma)=c(\omega) \leq h(M)-$ $m(\omega)$, which gives (3.5); similarly, Theorem 3.1 gives (3.6).

Example 3.6. For a torus $T^{n}$ and $S^{n} \times S^{1},(3.5)$ gives $M(\omega)+m(\omega) \leq|\operatorname{Sing} \omega|$; for $M_{g}^{2}, M(\omega)+m(\omega) \leq g+|\operatorname{Sing} \omega|-1$.

The estimates (3.5) and (3.6) are independent; cf. Remark 3.2 and the examples therein.

For a non-singular form, obviously, $M(\omega)+m(\omega)=1$. Note that for such forms, (3.5) does not hold, for instance, on $T^{n}$ and (3.6) does not hold, for instance, on $S^{n} \times S^{1}$

\section{Presence of minimal components AND HOMOLOGICALLY NON-TRIVIAL LEAVES}

A foliation without minimal components is called compactifiable. We will present some conditions for compactifiability of $\mathcal{F}_{\omega}$ and the presence of homologically nontrivial leaves in it (i.e., whether the foliation graph is a tree) in terms of rk $\omega$ and Sing $\omega$.

4.1. Conditions in terms of the rank of the form. Consider the form's rank $\operatorname{rk} \omega=\operatorname{rkim}[\omega]$, where $[\omega]: H_{1}(M) \rightarrow \mathbb{R}$ is the integration map. If $\omega$ is rational $($ rk $\omega \leq 1)$ then $\mathcal{F}_{\omega}$ is compactifiable, i.e. $m(\omega)=0$ [13]. We will show that the converse is true in the sense that any compactifiable foliation can be defined by a rational form; more specifically, the ranks of forms defining such a foliation cover a limited range starting from 0 or 1.

Recall that a maximal component $\mathcal{C}_{j}^{\max }$ is $\gamma_{j} \times(0,1)$ for some leaf $\gamma_{j}$ and $M(\omega)$ is the number of maximal components.

Theorem 4.1. Let $\mathcal{F}_{\omega}$ be a compactifiable Morse form foliation and $r \geq 1$. Then there exists a Morse form of rank $r$ defining the same foliation iff $r \leq c(\omega)$.

Whether there exists such a form of rank 0 depends on the structure of the directed foliation graph [4]. 
Proof. It is known that

$$
H_{1}(M)=\left\langle D\left[\gamma_{1}\right], \ldots, D\left[\gamma_{M(\omega)}\right], i_{*} H_{1}\left(M \backslash \bigcup_{j=1}^{M(\omega)} \mathcal{C}_{j}^{\max }\right)\right\rangle
$$

where $D$ is a Poincaré duality map (i.e., $D\left[\gamma_{k}\right] \cdot\left[\gamma_{l}\right]=\delta_{k l}$ ) and $i$ is the inclusion: indeed, the cycles $z \notin\left\langle D\left[\gamma_{i}\right]\right\rangle$ in Figure 5 can be moved out of $\bigcup \mathcal{C}_{i}^{\text {max }}$, cf. (2.1); see a detailed proof in [3].
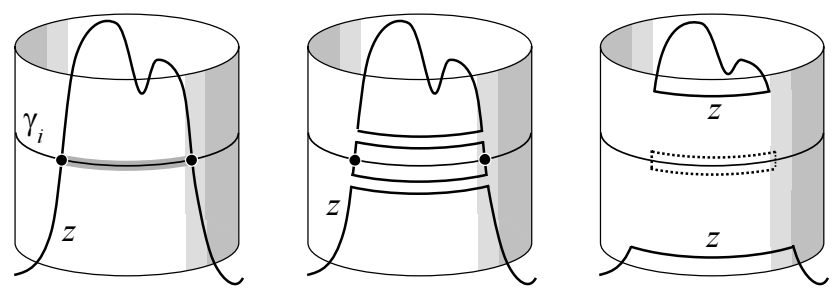

Figure 5. A cycle $z$ such that $z \cdot\left[\gamma_{i}\right]=0$ can be moved out of $\mathcal{C}_{i}^{\text {max }}$.

So rk $\omega$ is defined by $\left\langle D\left[\gamma_{k}\right]\right\rangle$, which by Theorem 2.1 has a basis $D\left[\gamma_{1}\right], \ldots, D\left[\gamma_{c(\omega)}\right]$. Let us realize each $D\left[\gamma_{i}\right]$ by a closed curve $\alpha_{i}$; by construction $\alpha_{j} \cap \mathcal{C}_{i}^{\text {max }}=\emptyset$ iff $i \neq j$. Without loss of generality we can suppose that $\alpha_{i} \cap \mathcal{C}_{i}^{\max }$ is connected and transverse to leaves.

Consider on $M$ a smooth function $f(x)>0$ constant on leaves in each cylinder $\mathcal{C}_{i}^{\max }$ and equal to 1 outside $\cup \mathcal{C}_{i}^{\max }$. The form $\omega^{\prime}=f(x) \omega$ defines the same foliation $\mathcal{F}_{\omega}$. By choosing an appropriate $f(x)$ we can vary its integrals along $\alpha_{i}$, $i=1, \ldots, c(\omega)$, obtaining any desired $\mathrm{rk} \omega^{\prime}$.

Example 4.1. The compactifiable foliation on $M_{g}^{2}$ (or $\sharp_{i=1}^{g} T^{n}$ ) shown in Figure 6 can be defined by a rational form with $\mathrm{rk} \omega=1$. It can also be defined by forms with $\operatorname{rk} \omega=2, \ldots, g$.

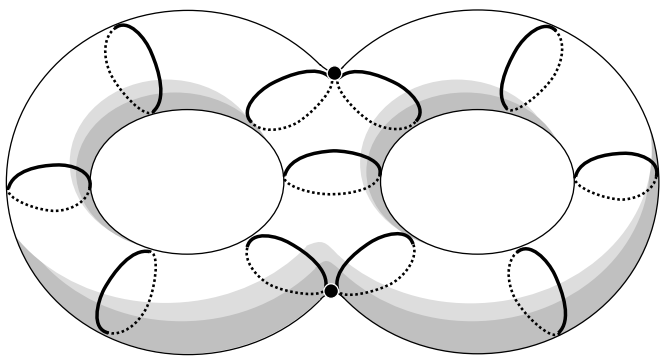

(a)

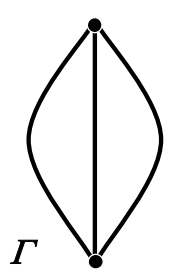

(b)

Figure 6. (a) The foliation from Example 4.1 and (b) its foliation graph. 
Corollary 4.1. For a compactifiable foliation $\mathcal{F}_{\omega}$ it holds $\mathrm{rk} \omega \leq c(\omega)$.

Corollary 4.2. Any compactifiable Morse form foliation can be defined by a rational form.

On the other hand, in any cohomology class with $\operatorname{rk} \omega>1$ there exists a form defining a minimal foliation [1]. What is more:

Corollary 4.3. If $\operatorname{rk} \omega>h(M)$ then $\mathcal{F}_{\omega}$ has a minimal component [3]. In particular, if $\mathrm{rk} \omega=b_{1}(M)$ (maximal) and the cup-product $\smile$ on $H^{1}(M, \mathbb{Z})$ is non-trivial then $\mathcal{F}_{\omega}$ has a minimal component.

Proposition 4.1. If $\operatorname{rk} \omega=b_{1}(M), H_{1}(M)$ has no torsion, and $\smile$ is nondegenerate, then $c(\omega)=0$, i.e., all compact leaves are homologically trivial.

Note that in these conditions, harmonic Morse forms have no compact leaves [2].

Proof. Suppose for some compact leaf $\gamma$ it holds $[\gamma] \neq 0$. We can construct a (nonMorse) form $\varphi$ trivial outside a cylindrical neighborhood of $\gamma$, in which $\mathcal{F}_{\varphi}=\mathcal{F}_{\omega}$; thus $\varphi \wedge \omega=0$. Since $[\gamma] \neq 0$, the form $\varphi$ can be chosen such that $[\varphi] \in H^{1}(M, \mathbb{Z})$. Thus $[\varphi] \smile_{\mathbb{R}}[\omega]=\sum \alpha_{i}\left([\varphi] \smile \xi_{i}\right)=0$, where $\smile_{\mathbb{R}}$ is the cup-product on $H^{1}(M, \mathbb{R})$ and $\xi_{i}$ is a basis in $H^{1}(M, \mathbb{Z})$. Since $H^{2}(M, \mathbb{Z})$ has no torsion, we have $[\varphi] \smile \xi_{i}=0$ for all $i$, which contradicts non-degenerateness of $\smile$.

4.2. Conditions in terms of the form's singularities. In Section 3, we have shown that $m(\omega)+c(\omega) \leq h(M)$. We will show that if there are more centers than conic singularities, then $m(\omega)+c(\omega)=0$, i.e., $\mathcal{F}_{\omega}$ is compactifiable and all its compact leaves are homologically trivial.

No necessary condition for compactifiability of $\mathcal{F}_{\omega}$ can be obtained in terms of Sing $\omega$ : for any $\omega$ there exists a rational Morse form with the same singularities with their indices $[14,15]$; its foliation is compactifiable. However, Sing $\omega$ can define the topology of $M$.

If $\operatorname{Sing} \omega=\emptyset$ then $M$ is a bundle over $S^{1}[15]$. the topology of $\mathcal{F}_{\omega}$ is defined by rk $\omega$ : if $\operatorname{rk} \omega=1$ then $\mathcal{F}_{\omega}$ is compact (all its leaves are compact), otherwise it is minimal.

If Sing $\omega \neq \emptyset$ but all its singularities are centers then $M=S^{n}$ and $\mathcal{F}_{\omega}$ is compact. We will generalize this fact: if there are more centers than conic singularities then $b_{1}(M)=0$.

Denote by $\Omega_{k}=\Omega_{k}(\omega), k \leq \frac{n}{2}$, the set of singularities of index $k$ and $n-k$. Let $\mu_{i}=\mu_{i}(\omega)$ be the number of singularities of index $i$, then $\left|\Omega_{k}\right|=\mu_{k}+\mu_{n-k}$ for $k \neq \frac{n}{2}$ and $\left|\Omega_{\frac{n}{2}}\right|=\mu_{\frac{n}{2}}$.

Theorem 4.2. If $\left|\Omega_{0}\right|>\left|\Omega_{1}\right|$ then $b_{1}(M)=0$.

Proof. (i) If $\left|\Omega_{0}\right|>\left|\Omega_{1}\right|$ then $\omega=d f$.

Indeed, there exists a rational Morse form with the same singularities and indices [14]; its foliation is compactifiable. Without loss of generality we can assume that each its leaf adjoins at most one singularity [6]. Thus its foliation graph $\Gamma$ has no vertices $P$ with $\operatorname{deg} P>3$; the singularity in any vertex with $\operatorname{deg} P=3$ belongs to $\Omega_{1}$, and the set of vertices with $\operatorname{deg} P=1$ is $\Omega_{0}$. 
For a connected graph, $2 m(\Gamma)=\sum(k-2) p_{k}+2 \geq 0$, where $m(\Gamma)$ is the cycle rank and $p_{k}$ is the number of vertices with $\operatorname{deg} P=k ; p_{1}=\left|\Omega_{0}\right|, p_{3} \leq\left|\Omega_{1}\right|$. Thus $\left|\Omega_{0}\right|>\left|\Omega_{1}\right|$ implies $m(\Gamma)=0$; by Theorems 2.1 and $4.1, \omega=d f$.

(ii) If $\operatorname{dim} M \geq 3$ and $\omega=d f$ then

$$
\left|\Omega_{0}\right|-\left|\Omega_{1}\right| \leq 2-2 b_{1}(M)
$$

Indeed, consider the Morse inequality

$$
F(\lambda)=\sum_{k=0}^{\lambda}(-1)^{\lambda-k}\left(\mu_{k}(f)-b_{k}(M)\right) \geq 0,
$$

which turns into equality for $\lambda=n$, where $n=\operatorname{dim} M$. Applying it to $F(1)+F(n-$ 2) $-F(n)$ gives

$$
-\left(\mu_{0}-b_{0}\right)+\left(\mu_{1}-b_{1}\right)+\left(\mu_{n-1}-b_{n-1}\right)-\left(\mu_{n}-b_{n}\right) \geq 0,
$$

which gives (4.1) since $\left|\Omega_{0}\right|=\mu_{0}(f)+\mu_{n}(f)$ and $\left|\Omega_{1}\right|=\mu_{1}(f)+\mu_{n-1}(f)$.

(iii) On $M_{g}^{2}$ it holds $\left|\Omega_{0}\right|-\left|\Omega_{1}\right|=2-b_{1}(M)$, which together with (ii) finishes the proof.

Now, Theorem 3.1 gives:

Theorem 4.3. If $\left|\Omega_{0}\right|-\left|\Omega_{1}\right|>0$ then $\mathcal{F}_{\omega}$ is compactifiable $(m(\omega)=0)$, all its compact leaves are homologically trivial $(c(\omega)=0)$, and the foliation graph is a tree.

\section{REFERENCES}

[1] P. Arnoux \&3 G. Levitt: Sur l'unique ergodicité des 1-formes fermées singulières. Invent. Math. 84 (1986), 141-156. Zbl 0577.58021

[2] M. Farber \& G G. Katz \& J J. Levine: Morse theory of harmonic forms. Topology 37:3 (1998), 469-483. Zbl 0911.58001

[3] I. Gelbukh: Presence of minimal components in a Morse form foliation. Diff. Geom. Appl. 22:2 (2005), 189-198. Zbl 1070.57016

[4] I. Gelbukh: Ranks of collinear Morse forms. Submitted.

[5] F. Harary: Graph theory. Addison-Wesley Publ. Comp., Massachusetts, 1994. Zbl 0182.57702

[6] K. Honda: A note on Morse theory of harmonic 1-forms. Topology 38:1 (1999), 223-233. Zbl 0959.58014

[7] H. Imanishi: On codimension one foliations defined by closed one forms with singularities. J. Math. Kyoto Univ. 19 (1979), 285-291. Zbl 0417.57010

[8] A. Katok: Invariant measures of flows on oriented surfaces. Sov. Math. Dokl. 14 (1973), 11041108. Zbl 0298.28013

[9] G. Levitt: 1-formes fermées singulières et groupe fondamental. Invent. Math. 88 (1987), 635667. Zbl 0594.57014

[10] G. Levitt: Groupe fondamental de l'espace des feuilles dans les feuilletages sans holonomie. J. Diff. Geom. 31 (1990), 711-761. Zbl 0714.57016

[11] I. Mel'nikova: A test for non-compactness of the foliation of a Morse form. Russ. Math. Surveys 50:2 (1995), 444-445. Zbl 0859.58005

[12] I. Mel'nikova: Maximal isotropic subspaces of skew-symmetric bilinear map. Vestnik MGU 4 (1999), 3-5. Zbl 0957.57018

[13] S. Novikov: The Hamiltonian formalism and a multivalued analog of Morse theory. Russian Math. Surveys 37:5 (1982), 1-56. Zbl 0571.58011

[14] A. Pazhitnov: The incidence coefficients in the Novikov complex are generically rational functions. Sankt-Petersbourg Math. J. 9:5 (1998), 969-1006. Zbl 0899.58009 
[15] D. Tischler: On fibering certain foliated manifolds over $S^{1}$. Topology 9 (1970), 153-154. Zbl 0177.52103

Author's addresses: I. Gelbukh, CIC-IPN, 07738, DF, Mexico.

e-mail: gelbukh@member . ams.org. 